\title{
Novel Product Development and Organoleptic Analysis Using Asparagus (Asparagus officinalis) Stems
}

\section{Jyoti D Vora ${ }^{1 *}$ and Rucha Khadke ${ }^{2}$}

${ }^{1}$ CEO, Dhirang Consultants, Mumbai, India

${ }^{2}$ Assistant Professor, Department of Biochemistry, VPM's B N Bandodkar

College of Science, Autonomous, Thane, India

*Corresponding Author: Jyoti D Vora, CEO, Dhirang Consultants, Mumbai, India.
Received: September 28, 2021

Published: October 27, 2021

(C) All rights are reserved by Jyoti D Vora and Rucha Khadke.

\begin{abstract}
Asparagus officinalis is consumed, owing to its richness of vitamins and minerals. Irrespective of its health benefits, Asparagus it is not widely used. The primary reason behind this is that, it is a highly perishable product. This study aimed at developing a Novel product and its organoleptic analysis in order to increase the shelf life of asparagus using age old and traditional method of food preservation. Acceptability of the product was analysed with semi trained panel of evaluators.
\end{abstract}

Keywords: Asparagus officinalis; Organoleptic Analysis; Novel Product Development; Sensory Evaluation

\section{Introduction}

Asparagus officinalis has been cultivated and harvested from the wild for thousands of years and has become an economically important crop. The common name asparagus derives from the Greek asparagos (and originally the Persian asparag) meaning sprout or shoot, referring to the succulent shoot tips (spears) that emerge in spring. The specific epithet officinalis means 'of the dispensary' in Latin, alluding to the medicinal properties of the plant. Chemical constituents of Asparagus officinalis include glycosides, flavonoids and the amino acid asparagine.

Therapeutic uses of Asparagus and its various health benefits, owing to its richness of vitamins and minerals are well known. Excellent provider of folate and balance it is known to rejuvenate the reproductive system in women. Rich in its potassium content, inclusion of Asparagus stems the depletion of calcium from our body. A good diuretic, it acts as a cleanser for the body removing toxic matter. Inulin in Asparagus consumes the bacteria in the large in- testine; thus, maintaining a healthy digestive system. Asparagus also possess antifungal, antiviral and anti-cancer properties [1].

The primary issues in the use of Asparagus widely is that, it is a highly perishable product. Its high respiratory rate necessitates the use of modified atmosphere packaging (MAP) has been used in order to increase the shelf-life of fresh asparagus $[2,3]$. Once trimmed and cooked, asparagus loses about half its total weight. Use asparagus within a day or two after purchasing for best flavour and texture. Store in the refrigerator with the ends wrapped in a damp paper towel.

The use of modified atmospheres and vacuum packaging to prolong the shelf-life of fruit and vegetables has always received a lot of attention Controlled atmosphere storage is used to prolong the storage and shelf-life of certain kinds of vegetables [4]. Lill [5] kept asparagus in non-perforated polyethylene bags for 8-35 days at $1^{\circ} \mathrm{C}$, obtaining good results. 
While MAP is a new age packaging solution and is receiving research interest, it has certain limitations like high complexity of the process, relatively high costs and effect on product quality. To overcome the shortfalls of MAP, this study aimed at developing a Novel product and its organoleptic analysis in order to increase the shelf life of asparagus and its sensory acceptance using age old and traditional method of preserving.

Organoleptic analysis (sensory evaluation) is a scientific discipline that applies principle of experimental design and statistical analysis to the use of human senses (sight, smell, taste, touch and hearing) for the purpose of evaluating products.

\section{Materials and Methods}

\section{Product development}

The process for increasing shelf life in this experiment was rooted in the traditional Indian way of food processing and preservation. Pickling is habitually carried out in Indian households to extend the longevity of vegetables and relishing them annually.

An edible product with Asparagus stems was made. It was based on traditional way or preserving vegetables and fruits by pickling in brine. Asparagus soaked for three months in brine, seasoned with garlic, vinegar, and spices which brings out the natural flavours of asparagus and increase shelf life. Its astringent flavour makes it a healthy and tasty meal accompaniment.

Hazard Analysis and Critical Control Point (HACCP) is an internationally recognized system for reducing the risk of safety hazards in food. HACCP Plans were prepared for each process during product development. Possible hazards includes biological, chemical or physical hazards were identified. Critical Control Points (CCP) were put in place to make sure the hazards are eliminated or controlled to ensure acceptable levels of quality in the food product.

In order to project organoleptic appeal of the asparagus, sensory evaluation was carried out with a semi trained panel. A panel comprising of bio-statistical significant number of panellists was chosen for evaluation.

\section{Assessment of organoleptic acceptability}

- $\quad$ The sample was analysed by visual, olfactory and gustatory response.

- $\quad$ Thorough sensory evaluation procedure involving semi- trained panellists. The panellists answered a detailed questionnaire based on several non-invasive and invasive sensory parameters.

- The non-invasive section of the questionnaire ascertained the awareness of the nutritional benefits of asparagus among panellists. The invasive section of the questionnaire focused on determining product acceptability, product value for money and its commercial marketability.

\section{Results and Discussion}

\section{Non-invasive analysis}

$97 \%$ of panelists agree that pickles form an important part of a delicious meal suggesting that pickles have been an integral part of a meal. 91\% panelist like pickled vegetables $91 \%$ panelist like pickled vegetables. Majority of the panelist consume pickled vegetables once in a week or on daily basis. Asparagus is consumed infrequently be majority $77 \%$ of the panelist. $94 \%$ of panelist agree that the idea of asparagus pickle is appealing. Majority of the panellist were unaware about the health benefits of asparagus.

\section{Invasive analysis}

The ratings of invasive analysis of colour, texture, flavour, appearance, etc. are ranging from 5-9 i.e. towards the higher side of the scale which ascertains the positive organoleptic acceptance of the product. Majority of panelist found the taste of the product to be vinegary or sour. $80 \%$ of panelist would like to consume the product again. $97 \%$ panelist say that the price is appropriate indicating high value for money of the product $[7,8]$.

\section{Conclusion}

The trends in the result of sensory evaluation confirm that there is minimum awareness about the health benefits of consumption of asparagus. It is evident that only a small number of people consume asparagus. Consumption of asparagus is a matter of chance and not by choice, as determined by analysis of questionnaire. The sensory evaluation done by panellist exhibits very high organoleptic acceptance of the product developed from asparagus. The value for money of the product was also found be high.

The product developed is a novel and convenient way for increasing the shelf life of asparagus. Moreover asparagus stems can be remove from brine, washed and be incorporated in other reci- 
pes which makes it versatile. Manufacturers thus need to exploit the dual benefits of versatility off asparagus.

\section{Bibliography}

1. Polo-Ma-Abiele., et al. "Medicinal Properties of Selected Asparagus Species: A Review” (2019).

2. Tenorio M., et al. "Changes in carotenoids and chlorophylls in fresh green asparagus (Asparagus officinalis L) stored under modified atmosphere packaging". Journal of the Science of Food and Agriculture 84 (2004): 357-365.

3. Everson, H., et al. "Effects of modified atmospheres on textural and cell wall changes of asparagus during shelf-life". International Journal of Food Science and Technology 27 (1992): 187199.

4. Benyathiar P., et al. "Shelf life extension of fresh asparagus using modified atmosphere packaging and vacuum skin packaging in microwavable tray systems". Packaging Technology and Science 33 (2020): 407-415.

5. Lill RE. "Storage of fresh asparagus". New Zealand Journal of Agricultural Research 8 (1980): 163-167.

6. Dipak Vora., et al. "Biochemical, Organoleptic and Antimicrobial Assessment of Asparagus (Asparagus Officinalis)". Journal of Biotechnology and Biochemistry 3.2 (2017): 83-94.

7. RM García-Gimeno., et al. "Determination of packaged green asparagus shelf-life". Food Microbiology 15.2 (1998): 191-198.

8. Jyoti D Vora and Padma Srinivasan. "An insight into organoleptic analysis of a novel product developed from lotus stem". International Journal of Food Science and Nutrition 1.5 (2016): 32-36.

Volume 5 Issue 11 November 2021

CAll rights are reserved by Jyoti D Vora and Rucha Khadke. 